\title{
Erratum to: Width of Sunspot Generating Zone and Reconstruction of Butterfly Diagram
}

\author{
V.G. Ivanov • E.V. Miletsky
}

Published online: 8 December 2010

(C) Springer Science+Business Media B.V. 2010

\section{Erratum to: Solar Phys}

DOI 10.1007/s11207-010-9665-6

During production, the final word of the title was omitted from the published article. The full and correct title of the article is

\section{Width of Sunspot Generating Zone and Reconstruction of Butterfly Diagram}

The online version of the original article can be found under doi:10.1007/s11207-010-9665-6. 